Mens

revue d'histoire intellectuelle de l'Amérique française

\title{
Louis Cornellier. Devoirs d'histoire. Des historiens québécois sur la place publique. Sillery, Septentrion, 2002. 131 p.
}

\section{Patricia-Anne De Vriendt}

Volume 5, numéro 1, automne 2004

URI : https://id.erudit.org/iderudit/1024396ar

DOI : https://doi.org/10.7202/1024396ar

Aller au sommaire du numéro

Éditeur(s)

Centre de recherche en civilisation canadienne-française

ISSN

1492-8647 (imprimé)

1927-9299 (numérique)

Découvrir la revue

Citer ce compte rendu

De Vriendt, P.-A. (2004). Compte rendu de [Louis Cornellier. Devoirs d'histoire. Des historiens québécois sur la place publique. Sillery, Septentrion, 2002. 131 p.]

Mens, 5(1), 181-186. https://doi.org/10.7202/1024396ar d'utilisation que vous pouvez consulter en ligne.

https://apropos.erudit.org/fr/usagers/politique-dutilisation/ 
et antiféministes), étant donné que bon nombre de caricaturistes et d'illustrateurs durant la période étaient des hommes.

Pour le moment, cette Anthologie constitue une " histoire par les textes » du féminisme québécois. La période couverte de même que le grand nombre de textes et d'auteures présentés permettent d'adopter une perspective globale autour du sujet. L'ouvrage est à la fois utile pour les étudiants et étudiantes de premier cycle et pour les chercheurs et chercheuses à la recherche de matériel «brut». On ne peut maintenant qu'espérer que l'appel de Micheline Dumont et de Louise Toupin, pour une synthèse d'histoire des idées féministes au Québec et pour leur meilleure intégration à la trame historique générale, sera entendu.

\author{
Amélie Bourbeau \\ Département d'bistoire \\ Université du Québec à Montréal
}

\title{
Louis Cornellier. Devoirs d'histoire. Des historiens québécois sur la place publique. Sillery, Septentrion, 2002. $131 \mathrm{p}$.
}

La lecture de Devoirs d'bistoire. Des historiens québécois sur la place publique de Louis Cornellier nous donne envie de retourner sur les bancs d'école, de préférence au Cégep de Joliette où enseigne l'auteur, également chroniqueur au Devoir. Le petit livre paru chez Septentrion rassemble quelque dixsept chroniques dans lesquelles Cornellier nous fait part des critiques et des réflexions que lui a inspirées la lecture d'ouvrages historiques québécois. Ce faisant et à son insu, il nous fait envier ses élèves... 
C'est avec un remarquable esprit de synthèse joint à une honnêteté et une générosité intellectuelles que Cornellier reconstitue l'arène des débats historiographiques québécois. La forme du propos nous convie à une sorte de pièce dont le scénario est un échange entre les Bouchard, Rudin, S. Gagnon, Trudel, Létourneau, Lester, pour ne nommer que ceux-là. Cornellier ne manque pas, en aparté, de nous faire part de ses points de vue. Par exemple, il s'inquiète de ce que la langue française telle que l'envisage Gérard Bouchard, c'est-à-dire dépouillée de sa charge identitaire, ne représente un dénominateur commun "réducteur, inutile et appauvrissant ", tout québécois et englobant fut-il. Cornellier remarque également que si l'appel de Serge Gagnon à dépasser la subjectivité, qui est un obstacle à l'avancement de la science, est compréhensible et même salutaire, il faut néanmoins garder à l'esprit que "le choc des visions assumées demeure la meilleure voie d'accès à une réalité toujours fuyante, celle de l'existence humaine ». Il affirme aussi que si on peut, à l'instar de Christian Dufour, critiquer la version écrite du Canada: une histoire populaire et trouver qu'elle fait la part belle aux vainqueurs de 1759, on ne saurait honnêtement oublier que l'intention au cœur de cette démarche s'accroche aux faits bruts davantage qu'à l'histoire des mentalités et aux interprétations sociologiques.

D'ailleurs, quelle incidence doit-on accorder à l'issue de la Conquête sur le devenir québécois ? Cette question est au centre d'un des textes de notre enseignant. L'ouvrage constitue donc, pour la forme, un " choc au sommet» des intellectuels québécois orchestré par un vulgarisateur et un pédagogue hors pair, amoureux du Québec, curieux de tout, passionné et engagé. En ce qui a trait au fond, l'auteur nous offre plusieurs actes, plusieurs thèmes selon les protagonistes en scène. Ainsi, il s'interroge sur ce que nous a laissé en héritage $L a$ famille Plouffe, dont le cinquantenaire est éclipsé par celui du 
Refus global. Il déplore la chasse aux sorcières menée par Esther Delisle. Il aborde la question de l'utilité de l'histoire telle qu'entendue par Georges Langlois, celle de la pertinence de l'histoire nationale et celle de l'expérience historique collective québécoise comme celle d'une collectivité neuve. Il traite enfin de l'apport historiographique des Normes de Maurice Séguin, de l'histoire monumentale de Marcel Tessier, c'est-àdire de l'histoire comme l'épopée de nos héros légendaires, de l'ambivalence identitaire québécoise, de l'héritage que nous a laissé la Révolution tranquille ainsi que de la façon dont on pourrait s'en montrer dignes sans en être dupes.

Étourdissante, éclatée, cette visite dans l'arène intellectuelle à laquelle nous convie Cornellier ? Peut-être au premier abord. Mais pour peu qu'il soit attentif, le néophyte saura rapidement trouver quelques précieux points de repère. Les chroniques sont présentées en ordre chronologique : on peut donc suivre la pensée de l'auteur au fil de ses lectures. Pour ce qui est du fond, des figures intellectuelles resurgissent périodiquement. Rudin, dont on sent très bien qu'il a la faveur de Cornellier, entre en scène à plusieurs reprises. En effet, à l'instar de notre critique érudit, l'historien de l'Université Concordia souligne à gros traits rouges la nature foncièrement polémique de l'historiographie, l'empreinte subjective que l'on trouve toujours, plus ou moins floue, sur le dire de l'historien. C'est pourquoi lorsqu'il est question d'histoire nationale, Rudin ne manque pas d'entrer en scène pour mettre en lumière les ressorts idéologiques que peut comporter une telle entreprise.

On le devine, l'omniprésence de Rudin s'explique aussi par le fait que les auteurs que Cornellier lit y font souvent référence, lui qui en avait égratigné plus d'un en 1998 avec son fameux Faire de l'bistoire au Québec. On retrouve donc dans les ouvrages dont rend compte Cornellier quelques-unes des foudres que s'est attirées Rudin en examinant la production 
historiographique québécoise. C'est le cas des Normes de Maurice Séguin, dans lequel Pierre Tousignant accuse Rudin d'avoir grandement sous-estimé l'apport historiographique de Maurice Séguin. C'est encore le cas du livre de Serge Gagnon intitulé Passé composé, dont l'objectif est de réfuter la thèse rudinienne. De plus, Cornellier fait appel au regard critique de Rudin pour étoffer sa réflexion. Par exemple, dans son compte rendu des Mythes et réalités dans l'bistoire du Québec de Marcel Trudel, Cornellier, citant Rudin, rappelle la tendance de Trudel à se pencher de façon autocritique sur les incidences de la Conquête. Enfin, Rudin revient tout au long des Devoirs d'bistoire parce que Cornellier partage ouvertement avec lui son " allégeance " à une approche polémique de l'histoire. Cette allégeance est explicite dans le compte rendu qu'il fait du débat opposant Christian Dufour et Mario Cardinal sur Le Canada : une bistoire populaire :

Tout travail historique, de dire Cornellier après avoir synthétisé la confrontation Cardinal-Dufour, met en jeu une dialectique faits-interprétations extrêmement complexe qui explique, pour reprendre la formule de Ronald Rudin, «la nature polémique de l'historiographie ». Accepter cette contrainte, qui est aussi un passionnant défi, c'est reconnaître le caractère inéluctäble des conflits d'interprétations et, par conséquent, le fait que l'argumentation, dans le champ historiographique, ne saurait se limiter à des slogans et à des jugements péremptoires. (p. 127)

Ainsi, pour maintes raisons Rudin réapparait toujours au détour de l'une ou l'autre des chroniques de Cornellier comme un fil conducteur entre différentes scènes de la vie intellectuelle québécoise.

Mais il ne s'agit pas de polémiquer pour polémiquer. Bien que Cornellier entretienne « des rapports troubles avec les prétentions scientistes de certains praticiens de ce que l'on 
appelle les sciences de l'homme ", on sent chez l'auteur, à défaut d'une quête de vérité dont l'issue le musellerait inévitablement dans une sorte de certitude idéologique, un amour certain de cette quête de vérité dont il se fait l'observateur critique. Cet amour de la quête, du questionnement perpétuel, que traduit une réelle volonté de compréhension des auteurs qu'il aborde, lui confère une générosité somme toute assez peu commune. Cela n'est nulle part plus évident que dans son rapport avec Gérard Bouchard. Après une première approche où il rechigne face à la thèse de La nation québécoise au futur et au passé qu'il qualifie de "nation building ", Cornellier nuance son jugement après la lecture subséquente du Dialogue sur les pays neufs, qui rend le projet bouchardien nettement " moins irritant ».

Cornellier laisse toujours la chance au coureur, sauf peut-être lorsqu'il s'agit d'Esther Delisle dont le Mythes, mémoire et mensonges ne semble pas avoir bénéficié de la même clémence que Le livre noir du Canada anglais de Normand Lester. Mise à part cette entorse à une règle certaine du bénéfice du doute, il y a chez Cornellier une générosité prédominante et, surtout, lucide. On aurait pu penser que Cornellier allait être prompt à juger Normand Lester, dont l'entreprise et le biais sont sans nuance, sans équivoque. Cornellier interroge pourtant : "peut-on dire que Lester dit des faussetés ? "C'est aussi avec une même générosité que notre chroniqueur aborde les propos de Lester et de Mario Cardinal, directeur de la publication du Canada: une histoire populaire. Peut-on trouver deux antagonistes plus éloignés sur le plan idéologique? Pour peu que les auteurs soient honnêtes envers les faits qu'ils étudient, Cornellier leur rend la pareille. C'est d'ailleurs ce qui explique à ses yeux que la chasse aux sorcières façon Esther Delisle ne passe pas la rampe de la probité intellectuelle. Encore une fois, il apparaît pertinent d'interroger les similitudes entre le type d'entreprise de Lester et celle de Delisle. Certes Lester 
ne dit pas des faussetés, mais ne mène-t-il pas lui aussi une enquête historique sur le mode de la chasse aux sorcières?

Il y a chez Cornellier un amour de la vérité en tant que recherche, une véritable quête de nuances, de compréhension et d'érudition qui le mène parfois, pour notre plus grand bonheur, en terrain inconnu. C'est ainsi qu'il nous convie à une agréable incursion dans l'histoire féministe façon Micheline Dumont, bien que, il s'en confesse, ce domaine d'intérêt ne soit pas «son fort». On ne peut que se réjouir de cette belle ouverture d'esprit!

Pour le plus grand bonheur de ceux qui n'ont pas toujours l'occasion de lire les chroniques du Devoir ou les livres de nos historiens, nous concluons par cet appel, le même en fait que lance Cornellier après sa lecture du Dialogue sur les pays neufs: "on en veut encore!"

Patricia-Anne De Vriendt Département d'bistoire Université Laval

Pierre Vallières. Paroles d'un nègre blanc. Anthologie préparée par Jacques Jourdain et Mélanie Mailhot, Montréal, VLB éditeur, 2002. 286 p. (Coll. " Parti pris actuels").

Indépendantiste, socialiste, révolutionnaire, felquiste, terroriste, péquiste, militant gai, internationaliste mais aussi chrétien. Ce sont tous des qualificatifs qu'on a attribués à Pierre Vallières (1938-1998) au cours de ses quarante années d'engagement sociopolitique. Cette énumération peut sembler de prime abord contradictoire mais, à la lecture de cette anthologie consacrée au révolutionnaire québécois, nous pou- 